\title{
Study on the Choreoecology of Hegang Dance, a Traditional Dance, in Yihuang, Jiangxi
}

\author{
$\mathrm{Shu} \mathrm{Hu}^{1, *}$ Fen $\mathrm{Liu}^{1}$ \\ ${ }^{1}$ Fuzhou Preschool Education College, Fuzhou, Jiangxi, China \\ "Corresponding author. Email: $48404932 @$ qq.com
}

\begin{abstract}
Yihuang Hegang dance, which enjoys a history of more than 600 years, refers to a traditional folk dance developed by generations of mountain people on their way up the mountain to cut firewood using hegang (rice straw) and chopper to play the rhythm of song and dance. Through the field investigation in Yihuang, Jiangxi Province, the ecological environment of Yihuang Hegang dance was explored, and its characteristics in different historical periods were analyzed. The research on the characteristics of the music, dance and costume shows that the inheritance and development of the dance cannot be separated from the dance elements in line with contemporary aesthetics.
\end{abstract}

Keywords: Hegang dance, Music, Dance, Ecology, Protection.

\section{INTRODUCTION}

As Yihuang locates in mountainous area, local people rely on firewood and hegang (rice straw) for fuel. Reflecting the wisdom and creativity of local people, Hegang dance was included in the fourth batch of national intangible cultural heritage list in 2014. Welcomed by Yihuang in Jiangxi Province, Hegang dance is a dance with strong local characteristics developed by the local working people in the way of chopping firewood using their choppers and hegang (rice straw) to match the beat of folk songs.

\section{ECOLOGICAL ENVIRONMENT THAT NOURISHES HEGANG DANCE}

Yihuang County, located in the east of central Jiangxi Province and the south of Fuzhou City, or the transition zone from Wuyi Mountains and Yuedu Mountains to Fuhe Plain, belongs to Fuzhou City of Jiangxi Province. The county covers an area of 1,944 square kilometers and has jurisdiction over 12 townships, 2 reclamation farms and 1 provincial industrial park, enjoying subtropical monsoon

*Fund: This research is a phased result of "Study on Hegang Dance in Yihuang, Jiangxi", a university humanities and social science research project of Jiangxi Province in 2020. (Project No. YS20109) humid climate. It boasts long history, rich products, large mountainous area, and various wild plants. "八 山半水一分田，半分道路和庄园 (most of the local terrain is mountain, a small part is water and fields and road manor)", a saying of local people, well depicts Yihuang's geographical picture. Yihuang is a key county of forestry in Jiangxi Province, with an area of 2,263,000 mu of mountain forest. Such a beautiful land of Yihuang has nourished colorful folk art, including four types of music and dance with local characteristics: Hegang dance, Nuo dance, Yihuang opera, and Yanghua opera. Among them, Hegang dance and Yihuang opera are national intangible cultural heritages.

Hegang dance can be found in Yihuang county and surrounding areas of Jiangxi Province, covering 12 towns and villages under the jurisdiction of counties: Taopi, Lixi, Fenggang, Tangyin, Nanyuan, Erdu, Zhenkou, Zhonggang, Shengang, Huangpi, Dongpi, and Xinfeng. It refers to a type of dance with strong local flavors developed by the local working people in the way of chopping firewood using their choppers and hegang (rice straw) to match the beat of folk songs.

Originally Hegang song, Hegang dance finally takes shape thanks to the contribution and artistic processing of local working people. The rhythm of Hegang song is smooth and brisk. Simple 
performance involving chopper and hegang is added when people sing the song. The range of movements required is moderate and the number of people is unlimited. When dancing, hegang is held in the hand or shoulder, and knocked by each part of the chopper to match the beats of dance and song. The steps are natural, mostly measured steps. Hegang dance enjoys a history of about 600 years.

\section{THE EVOLUTION OF HEGANG DANCE}

\subsection{The Embryonic Stage: Folk Songs}

Hegang dance is presented in the form of folk songs in the early stage, because then the local people used firewood as fuel, and chopper and bamboo as the labor tools for cutting and picking firewood. On the way up the mountain to cut firewood, people hold sickles in their hands and use the head to play the rhythm on the hegang to make sound and sing in concert with folk songs. At first, they sang Zhuo Wang Shan to the rhythm of the melody. They sang and beat by themselves, or divided jobs, improvising the words. Lyrics or borrow scenery lyric fun guessing, or love, or curse. As for the lyrics, some express feelings for fun and riddles through view borrowing, while others are employed to talk about love or curse. This is the earliest prototype of Hegang dance.

There are lush pine trees on Zhuo Wang Mountain.

I resort to folk songs to express my feeling.

I am looking forward to the lover who has been long overdue.

How can I let you know what I want.

Zhuo Wang Shan

\subsection{Development Stage: Hegang Song}

With its unique form, it won the favor of men and women, old and young, who will sing a few words whenever they go up the mountain to cut firewood together. They compete in groups or in chorus, singing melodious and ardently. People who were keen on innovation began to experiment with different percussion movements, beginning with a simple hegang in hand or on the shoulder and a single percussion beat with a chopper. Innovators not satisfied with such single form of performance also began to improve the performance movements, using different positions of chopper to touch the hegang, thus producing different timbres and tones. Such move enriches the rhythm and hand performance of hegang song, makes the song more vivid and lively. Such art form of singing while hitting the hegang is called "hegang song".

Heegang song is a folk song of low tone (a style of music with a short range, orderly couplets, regular rhythm, no drawl, and close to minor key), among which the most widely circulated and the most representative one is Zhuo Wang Shan ("Figure 1"). The music structure of hegang song includes four-tone series represented by 3216 and 3561 and three-tone series represented by 561 and 123, among which the former is the most representative. Most of the music follows couplet structure, with a free voice and a smooth rhythm. The $5 / 8$ beat and $4 / 8$ beat are commonly used to match the different parts of the chopper to hit hegang, and light and dexterous hand dance movements are made, fully showing the lively local style and traditional folk customs. Examples of music score of Hegang dance:



Figure 1 Structure: a verse (stanza).

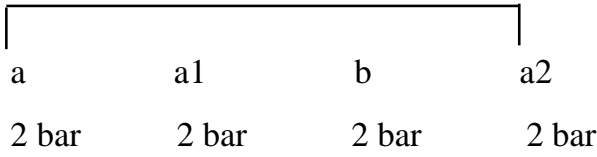

backbone tone: "3 2 1"

B flat

Zhuo Wang Shan is the most recognizable piece of hegang dance music. It is a tune with the style of a folk song, named after Zhuo Wang Mountain. It is a verse song composed of 4 phrases, and each 2 bars is a phrase. It's a square piece, $5 / 8$ beat, B flat. This work does not have a wide range, the highest note and the lowest note are five degrees apart. The whole song revolves around the three backbone notes "3, 2 and 1", which are mainly developed in a progressive way. The intonation of each sentence of the work is on "3-3-1-3", and the melody falls on 
"2-1-2-1". In terms of melody, it takes "3" as the central tone and develops with arched melodic lines through the combination of three-tone series. As for the overall direction of the melody, the first two phrases pitch from high to low ("3-1"), while the last two phrases form an arched melodic line ("1-3$1 ")$. The composition of the melody of the four phrases plays the role of "opening, developing, changing and concluding". Zhuo Wang Shan is often sung when people go up the mountain to cut firewood, with chopper beating hegang as an accompaniment, mostly for male and female duet. $\mathrm{XX}-\mathrm{XX}-\mathrm{XX}-\mathrm{X}$ is a common beat rhythm.

\subsection{Forming Stage: Hegang Dance}

The performance of Hegang dance has also made great progress with the continuous development of Hegang song. Some performers banged on the hegang, sang and danced, and experimented with various forms of duet. Some other performers tried the formation in the performance, inserted the bars on the ground to enrich the performance, and formed a natural marching action. The dance requires a small range of movements, mostly square steps, and there are no restrictions on participants, highlighting the characteristics of life and labor. After long-term development, the artistic style of "Hegang dance" was finally formed. In 2009, Hegang dance was listed as one of the first county-level intangible cultural heritage dance projects. In 2013, it was approved as a provincial intangible cultural heritage dance project. In 2014, it was listed in the fourth batch of national intangible cultural heritage dance projects.

\section{THE MUSIC CHARACTERISTICS OF HEGANG DANCE}

Hegang dance music belongs to folk song of low tone. $5 / 8$ beat and 4/8 beat are often employed in such traditional music. It enjoys free voice, fixed singing method, percussion sound through chopper and hegang which is deep and magnificent. The music structure of hegang song includes four-tone series three-tone series. The lyrics fall into three camps, namely love songs, lock songs (also riddles) mostly for male and female duet, and abusive song (lyrics not included).

As for the music structure, 123 that borrows from bi-xing (parable and elation) is adopted for singing love songs. 561 is employed for lock songs that emphasizes set up eight riddles and solve them one by one. Unlike a set melody, the lyrics tend to be improvised, depending on the actual situation.

\subsection{The Dance Characteristics of Hegang Dance}

The performance of Hegang dance combines singing and dancing, creating a lively and rich atmosphere. It was included in the Collection of Chinese Ethnic and Folk Dance · Jiangxi Volume in the 1980s. As an independent dance, it enjoys not only unique musical beat, but also special performance props. Different from most traditional dances in China, which take fans, silk and flowers as performance props, it uses the tools of people's labor, namely, hegang and sickles, as the performance props. In addition, performance props can also be adopted as dance instruments to beat the rhythm.

The dance movement is mainly composed of three: hand percussion movement, hegang position change movement and foot marching movement. The hand percussion movement has experienced the transformation from the initial simple percussion of hegang with the chopper in hand, to the varied percussion of hegan with the front and back of chopper, and the groove that connecting the handle and the chopper while matching the rhythm of the folk song. As for the change of the hegang position, the hegang was thrust into the ground at an angle, carried on shoulders or hands for rotation. The common ones include percussion bars, female singing and male percussion, male singing and female percussion, and stop percussion bars after singing a paragraph of lyrics. The marching movements of Hegang dance include general marching, single-span horse and double-span horse. During the performance, they sometimes advanced, sometimes backward, sometimes cross, imitating the movement of walking on the mountain path.

During the performance, these three movements will be integrated. "杜杠咯杠咯", the most common hand beat, coupled with $5 / 8$ beat, prepares ground for movements including forward strike bar, backward strike bar, lunge strike bar, up and down cutting bar, single-span horse strike, double-span horse strike, and jump swing sickle. The Hegang dance performance is not limited by the venue and the number of people. All people covering men and women, old and young engaged in singing and dancing every holiday celebrations, which is lively and simple. 


\section{THE COSTUMES AND PROPS OF HEGANG DANCE}

\subsection{Costumes}

The costumes of Hegang dance are very exquisite. Men wear white shirts with front opening, blue slacks with legs rolled up to the knee, black cloth sandals, and tie a red cloth belt. Women wear daily clothes of printed cloth, blue slacks with legs rolled up to the knee, black cloth bib and black sandals. These costumes feature strong national flavor. Dancers in simple but well-dressed costumes will perform their favorite Hegang dance with gusto at every festival.

\subsection{Props}

The props consist of hegang (rice straw) and chopper. At first, the choppers used as props were all real choppers with cutting edges. However, given the frequent performances and safety reasons, the choppers without cutting edges are adopted. During a rehearsal in 2005, the blade head broke away and almost hurt people, so the original metal chopper head was replaced by wood. The $50 \mathrm{~cm}$ wood chopper has been adopted since then.

As a prop, hegang has always been a labor tool. Both ends are pointed and convenient to pick firewood. It has always been used as props. However, the popularity of Hegang dance and the participation of younger children have prompted people to resort to hegang with round ends, which hedges risk. In addition, the length has changed from 1.6 meters to 1.4 --1.6 meters, depending on the height of the actor. The improved props are more scientific and convenient to use.

\section{CONCLUSION}

Hegang dance, the wisdom crystallization of generations of working people, refers to a kind of art form that the working people create in the process of labor for daily life entertainment. The progress of science and technology and the change of human life style lead to the dilemma of no successor facing some excellent traditional music. How to realize the integration of contemporary aesthetic art into traditional dance art, thus carrying forward the innovation based on inheriting the tradition, is an issue concerning every dancer.

\section{AUTHORS' CONTRIBUTIONS}

Shu $\mathrm{Hu}$ collected the data for the paper and wrote the thesis; Fen Liu contributed to revising and editing.

\section{REFERENCES}

[1] Tian Qian. Introduction to Watershed Anthropology [M]. People's Publishing House, 2018. (in Chinese)

[2] Zi Huajun. Choreoecology [M]. Culture and Art Publishing House, 1991. (in Chinese)

[3] Xu Mengfei. Hegang Dance: The Art of Dancing in Work [N]. Chinese Culture Daily, 2014. (in Chinese)

[4] Xu Yumo. Yihuang County Annals [M]. Yihuang County Chronicles Compilation Committee, 1993. (in Chinese)

[5] Lan Fan. On Intangible Cultural Heritage Dance(Part One) [J]. Ethnic Art Studies, Issue 5, 2019. (in Chinese)

[6] Yu Ping. Dance Morphology [M]. Beijing Dance Academy Press. (in Chinese)

[7] Zhong Jingwen. Introduction to Folklore [M]. Shanghai Literature and Art Publishing House, 1998. (in Chinese)

[8] Ouyang Xiwei. Exploring the creation of dance repertoire with the theme of "intangible cultural heritage" [D]. Beijing Dance Academy, 2018. (in Chinese)

[9] (United States) Rudolf Arnheim. Art and Visual Perception [M]. Sichuan People's Publishing House, 1998. (in Chinese)

[10] Chinese Dance History-Jiangxi Volume [M], Academic Press, 2001. (in Chinese) 